\title{
Analysis and Research on the Centimeter Band Receiver Amplitude Calibration Method
}

\author{
Kai Wang $\mathbb{D}^{1,2,3}$ Maozheng Chen, ${ }^{1,2,3}$ Jun Ma, ${ }^{1,2,3}$ Xuefeng Duan, ${ }^{1,2,3}$ Yang Wang, ${ }^{1,2,3}$ \\ Liang Cao, ${ }^{1,2,3}$ Hao Yan, ${ }^{1,2,3}$ and Binbin Xiang $\mathbb{D}^{1,2}$ \\ ${ }^{1}$ Xinjiang Astronomical Observatory, Chinese Academy of Sciences, Urumqi 830011, China \\ ${ }^{2}$ Key Laboratory of Radio Astronomy, Chinese Academy of Sciences, Nanjing 210008, China \\ ${ }^{3}$ Xinjiang Key Laboratory of Microwave Technology, Urumqi 830011, China
}

Correspondence should be addressed to Kai Wang; wangkai@xao.ac.cn

Received 19 March 2020; Accepted 2 June 2020; Published 3 July 2020

Academic Editor: Michael Kueppers

Copyright (c) 2020 Kai Wang et al. This is an open access article distributed under the Creative Commons Attribution License, which permits unrestricted use, distribution, and reproduction in any medium, provided the original work is properly cited.

The receiver is a signal receiving device in a radio telescope system. As an important parameter to characterize the receiver performance, noise temperature is very practical to calibrate accurately. The traditional receiver noise temperature calibration method is the cold and ambient load method. Through the establishment of K-band ambient receiver, and its amplitude calibration test platform of the cold and ambient load method, chopper wheel method, and ambient and hot load method, comparison and analysis of the above three methods were carried out. The test and calculation results show that the test accuracy of the cold and ambient load method is about $1.3 \%$, that of the chopper wheel method (nonlow elevation) is about 3\%, and that of the ambient and hot load method is about 9\%. The test accuracy of the ambient and hot load method is slightly lower than that of the above two methods. The analysis is mainly due to the uncertainty of the hot load temperature and the small temperature difference between the two loads, which leads to the deterioration of the overall accuracy. But the advantage is that the method can perform real-time calibration in the process of observation, and it is easier to implement than the traditional cold and ambient load method. The results of noise temperature measurement are compared with those of theoretical calculation, the error is basically within $10 \%$, and it can satisfy the demand of the noise temperature test. In the future, we expect that on the basis of increasing the hot load temperature, further experiments were carried out on the thermostatic treatment of hot load and the accuracy of temperature acquisition, and finally we hope that this method can better meet the testing requirements of receiver noise temperature and radio source amplitude calibration.

\section{Introduction}

Radio astronomy is an observational subject that uses radio telescope to receive microwave signals from radio sources [1]. The microwave receiver is the receiving equipment specially used to receive radio signal in the radio telescope system [2]. The radio signal is reflected and focused by the radio telescope to phase center of the feed [3], and it is then transmitted to the receiver through the low noise amplifier (LNA) and filter, after mixing with the local oscillator (LO) signal and other related processing, and the intermediate frequency (IF) signal is finally transmitted to the data terminal and processed [4]. The composition of the receiver system is shown in Figure 1.
The purpose of receiver amplitude calibration is to set up a temperature scale, which can convert the response of radio source from the receiver into an equivalent temperature value, and finally combine with the antenna effective area, and efficiency can finally get the flux density of the radio source [5]. The general method is to obtain the response ratio $g$ (equation (1)) of the amplitude and temperature of a receiver by using the cold and ambient load method. Thereinto, $V_{\mathrm{amb}}$ is the power output of ambient load, $V_{\text {cold }}$ is the power output of cold load, $T_{\mathrm{amb}}$ is the temperature of ambient load, and $T_{\text {cold }}$ is the temperature of cold load.

$$
g=\frac{V_{\mathrm{amb}}-V_{\text {cold }}}{T_{\mathrm{amb}}-T_{\text {cold }}} .
$$




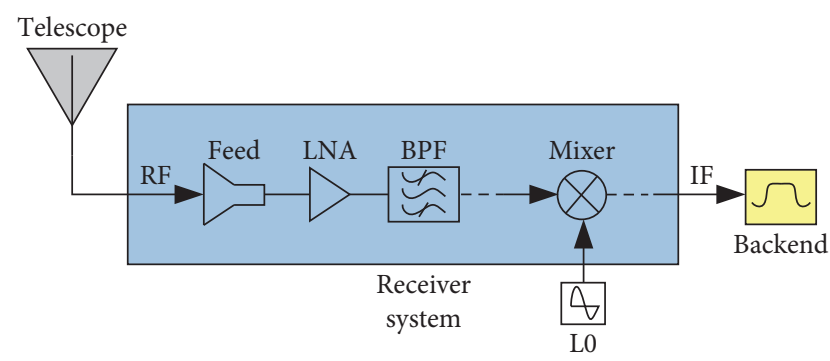

Figure 1: Signal transmission link of receiver.

Combined with this amplitude and temperature response ratio $g$, we can control the telescope to point on the radio source and point off, and then, we can get the equivalent temperature value of the radio source $T_{\mathrm{A}}$ (equation (2)). Thereinto, $V_{\mathrm{ON}}$ is the power output of pointing on the source, and $V_{\mathrm{OFF}}$ is the power output of pointing off the source.

$$
T_{A}=\frac{V_{\mathrm{ON}}-V_{\mathrm{OFF}}}{g} .
$$

Finally, the flux density $S_{\mathrm{V}}$ can be calculated by equation (3); thereinto, $\eta_{\mathrm{A}}$ is the antenna efficiency, $A_{p}$ is the antenna effective area, and $\mathrm{k}$ is the Boltzmann constant.

$$
S_{V}=\frac{2 \kappa T_{A}}{\eta_{A} A_{p}} \text {. }
$$

As the most core signal receiving equipment of the radio telescope system, the most important index of the receiver is sensitivity. Noise temperature is usually used to characterize the sensitivity of the receiver system, and higher sensitivity means that the receiver can detect weaker radio signals. Generally, the thermal noise generated by the receiver itself is called the noise temperature of the receiver, which is the equivalent temperature value of the amplitude response of the receiver after calibration.

In order to reduce the noise temperature of the receiver, the first stage amplifier with lower noise coefficient is usually used in the design of the centimeter band receiver. On this basis, if the sensitivity is expected to be further improved, it is generally used to cool the low noise amplifier and its prestage microwave devices (partly or wholly) below $20 \mathrm{~K}$ to reduce the noise temperature of the whole receiver system. Figure 2 shows the interior photos of dewar in a K-band cryogenic receiver of Xinjiang Astronomical Observatory (XAO).

In view of the fact that the noise temperature calibration of the receiver is the basis of the amplitude calibration of the whole telescope system and the key process to characterize the performance of the receiver, it is of great practical significance to carry out relevant calibration research in the corresponding band.

\section{Amplitude Calibration Method}

From the view of engineering, the most commonly used method of centimeter band receiver amplitude calibration is the cold and ambient load method, which is the traditional

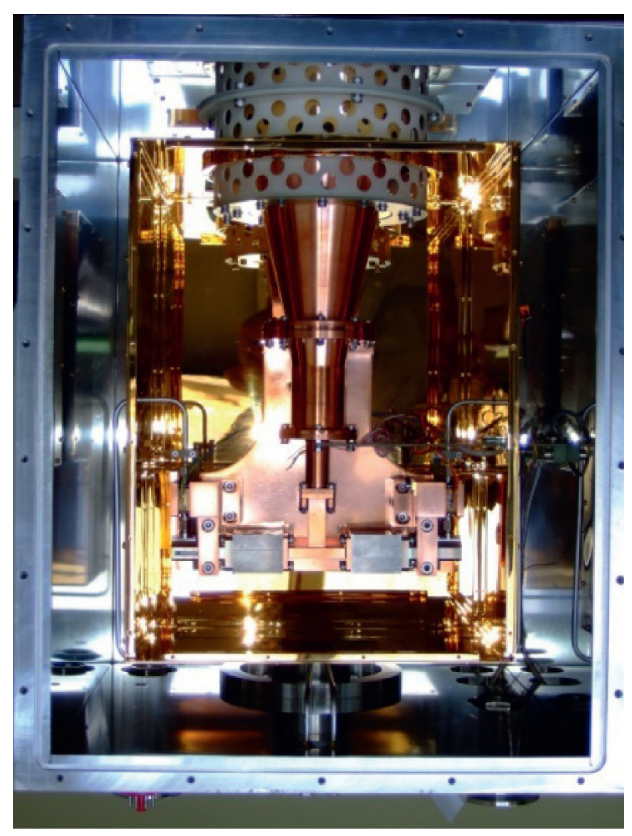

FIGURE 2: K-band cryogenic receiver of XAO.

low temperature and normal temperature load method. For the receiver with large aperture feed, for example, L-band receiver, because it cannot provide a large enough cold load to cover the feed interface completely, the chopper wheel method is generally used for calibration.

2.1. Cold and Ambient Load Method. As the most classical method in amplitude calibration, the cold and ambient load method is also the traditional method; according to the Planck blackbody radiation principle, any object has certain external heat radiation at different temperatures, and the radiation amplitude and the object temperature are approximately linear within a certain range [6]. The cold and ambient load method is just evolved based on this principle. Specifically, in the noise temperature test of the receiver, two wideband blackbody radiation sources with different physical temperature (such as the cold blackbody load in $80 \mathrm{~K}$ liquid nitrogen and the ambient blackbody load in $300 \mathrm{~K}$ room temperature) are used, and they are, respectively, placed on the feed interface of the receiver (the front stage of the first stage amplifier or mixer), so that the blackbody load can not only absorb the outside of the feed but also the radiation of the blackbody load can also be injected into the receiver to calibrate the noise of the receiver. The test of cold and ambient load method is shown in Figure 3.

We can directly get the receiver noise temperature, $T_{\text {rec, }}$ calculated by the cold and ambient load method from equation (4). After the noise calibration of the receiver is completed, the radio telescope is used to observe the radio source for pointing on or off, then the equivalent temperature value of the radio source can be obtained, and then, the flux density of the radio source can be obtained by combining the antenna efficiency and aperture. 


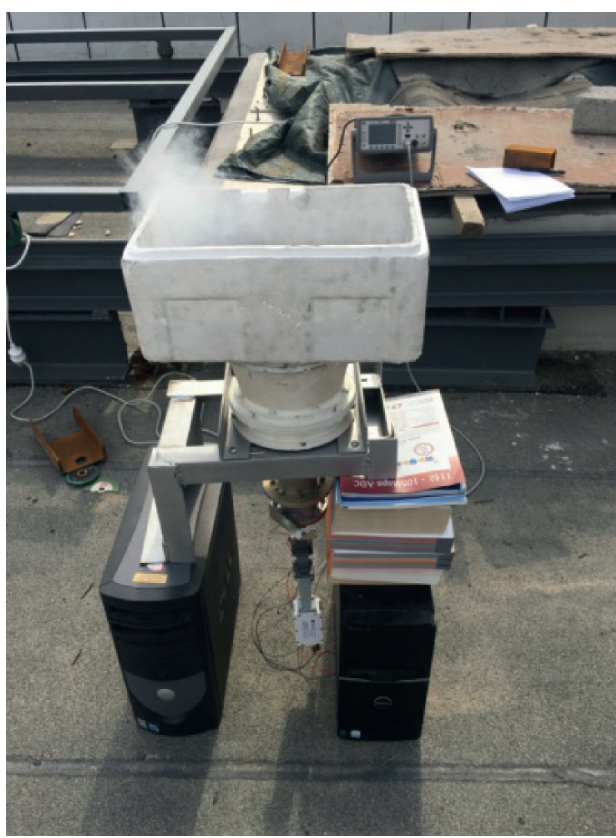

(a)

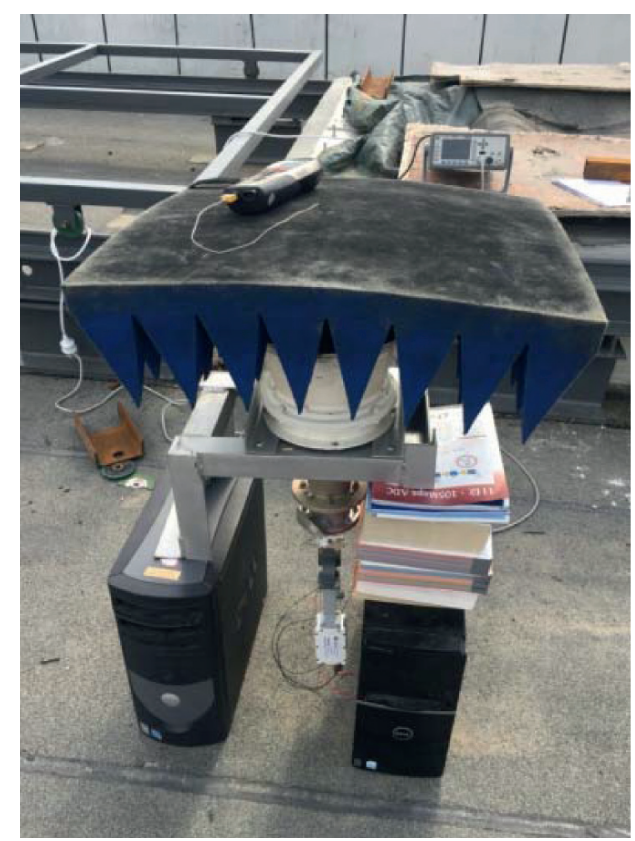

(b)

FIgURE 3: Ku-band receiver of test with the cold and ambient load method.

$$
T_{\text {rec }}=\frac{T_{\mathrm{amb}}-\left(V_{\mathrm{amb}} / V_{\text {cold }}\right) T_{\text {cold }}}{\left(V_{\mathrm{amb}} / V_{\text {cold }}\right)-1 .}
$$

Although the cold and ambient load method is a common method of amplitude calibration nowadays, it also has some limitations, which is mainly manifested in that when the aperture of the receiver's feed is large; it is difficult to carry out accurate noise temperature calibration because it is impossible to provide a vessel large enough to hold $80 \mathrm{~K}$ liquid nitrogen at the antenna end; it means that is impossible to provide the cold load after the receiver is installed in the antenna [7]. Figure 4 shows the cold load test of the ultra-wideband (UWB) receiver in the laboratory.

2.2. Chopper Wheel Method. The chopper wheel method is a method to test the noise temperature of the receiver by alternately introducing and removing an ambient load at the top of the feed, so that the receiver can alternately test the radiation of the ambient load and the sky [8-10]. The principle of this method is the same as that of the cold and ambient load method, but the cold load is replaced by cold sky. The noise temperature of the receiver can be measured only by introducing the ambient load during calibration. Equation (5) is the calculation formula of the chopper wheel method to calculate the receiver noise temperature, where $V_{\text {sky }}$ is the power output of the receiver beam receiving the cold sky at the current elevation, and $T_{\text {sky }}$ is the sky brightness temperature value of the corresponding frequency band at the current elevation [11-13].

$$
T_{\text {rec }}=\frac{T_{\mathrm{amb}}-\left(V_{\mathrm{amb}} / V_{\mathrm{sky}}\right) T_{\mathrm{sky}}}{\left(V_{\mathrm{amb}} / V_{\mathrm{sky}}\right)-1} .
$$

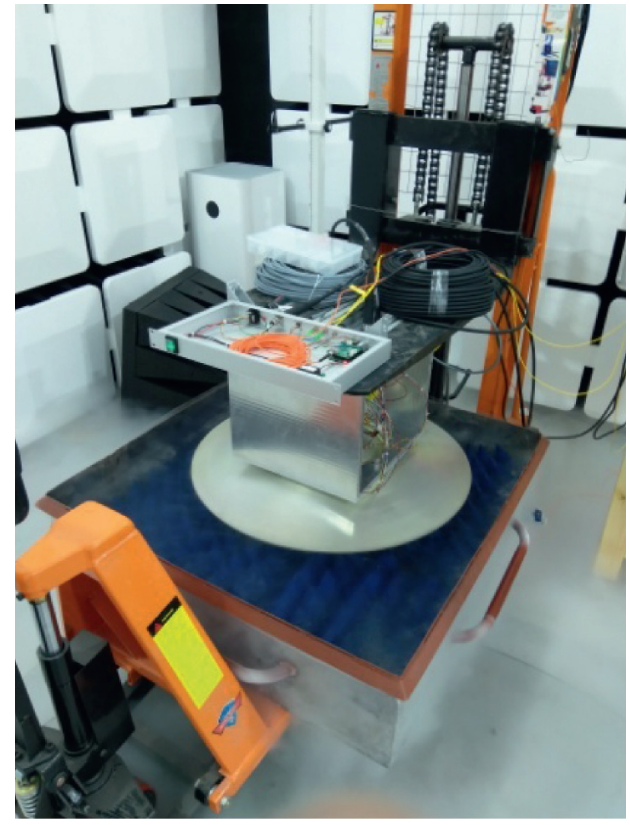

FIGURE 4: Cold load test of the UWB receiver.

The advantage of the chopper wheel method is that it can carry out real-time calibration when the antenna is in any elevation angle during observation, but it is necessary to know the brightness temperature of the sky in the corresponding frequency band and at different elevation angles. The calculation of sky brightness temperature $T_{\text {sky }}$ is shown in equation (6), $\eta$ is the antenna efficiency, $T_{\text {atm }}$ is the average temperature of the atmosphere in the vertical direction, $T_{\text {spill }}$ is the effective spillover temperature of the antenna beam, 
$T_{\mathrm{CBR}}$ is the cosmic background radiation (about $2.7 \mathrm{~K}$ ), and $\tau$ is the atmospheric opacity $[14,15]$.

$$
T_{\text {sky }}=\eta T_{\text {atm }}\left(1-e^{-\tau}\right)+(1-\eta) T_{\text {spill }}+\eta T_{\mathrm{CBR}} e^{-\tau} .
$$

Because the chopper wheel calibration is simple and easy, only ambient load with chopper function is required to be set on the feed interface of the receiver. During the calibration, only the sky brightness temperature value under different elevation angles of the corresponding frequency band is combined to control the ambient load to cover or remove the feed interface. Because the change of atmospheric absorption can be reflected in $T_{\text {sky }}$, the chopper wheel method is widely used in millimeter band amplitude calibration [16, 17]. As shown in Figure 5, it is the chopper wheel calibration system of IRAM.

\section{System Construction and Test}

In order to further analyze the centimeter band receiver amplitude calibration method, we need to build a centimeter band ambient receiver and its amplitude calibration platform to develop the research and verification of the abovementioned amplitude calibration methods.

3.1. K-Band Ambient Receiver. We have built a K-band ambient receiver in the laboratory, which is mainly composed of a feed, waveguide coaxial converter (WCC), LNA, bandpass filter (BPF), mixer, LO, IF amplifier, and other devices, as shown in Figure 6. The RF working bandwidth of the receiver is $22-24.2 \mathrm{GHz}$, and the superheterodyne design is adopted. The RF signal is amplified, filtered, and mixed, and the final output is $3.95-6.15 \mathrm{GHz}$ IF signal. In order to reduce the cost, only the left polarization signal of an orthomode transducer (OMT) is processed, and the relevant power acquisition is carried out, while the right polarization signal from the OMT is directly connected to the matching load after the wave to wave converter. All the test data after this paper are also from left polarization signal (IF1).

The picture of the K-band ambient receiver is shown in Figure 7. The low-noise amplifier and its subsequent microwave devices are installed on an aluminum plate, which not only helps to fix the whole on the back of the feed network, but also facilitates the heat dissipation of each active device.

Because the noise temperature of the receiver system mainly depends on the insertion loss of the passive device in the front link of the receiver and the noise and gain of the active device, gain GA can be calculated by equation (7); thereinto, $\mathrm{L}$ is the insertion loss of the whole link, $P_{\mathrm{i}}$ is the input power, and $P_{\mathrm{O}}$ is the output power.

$$
G_{A}=\frac{1}{L}=\frac{P_{\mathrm{o}}}{P_{\mathrm{i}}} \text {. }
$$

The gain of passive devices can be calculated from the above formulas by equation (8):

$$
G_{A}=\frac{1}{10^{(L(\mathrm{~dB}) / 10)}} .
$$

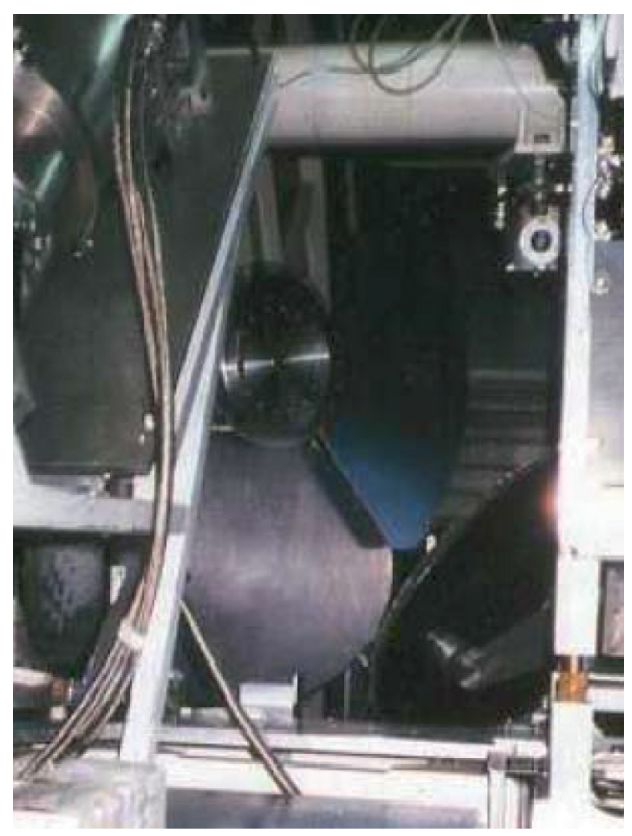

FIGURE 5: The chopper wheel calibration system of IRAM.

So the noise temperature $T_{\mathrm{r}}$ expression of the cascade system can be calculated by equation (9):

$$
T_{r}=T_{r 1}+\frac{T_{r 2}}{G_{A 1}}+\frac{T_{r 2}}{G_{A 1} G_{A 2}}+\cdots .
$$

Noise figure is an important parameter for many microwave devices [18], the noise figure of LNA for the K-band ambient receiver is $3 \mathrm{~dB}$. As shown in Table 1, the theoretical calculation of noise temperature for the K-band ambient receiver is $406.5 \mathrm{~K}$ when the ambient temperature is $295 \mathrm{~K}$.

3.2. Test of the Cold and Ambient Load Method. On the basis of the K-band ambient receiver, we built a test platform of the cold and ambient load method. The K-band test platform and cold blackbody load are shown in Figure 8.

The amplitude output adopts the total power test method to obtain the power. The power meter is Agilent N1914A, and the power probe is $\mathrm{E} 9300 \mathrm{~A}(10 \mathrm{MHz}-18 \mathrm{GHz})$, as shown in Figure 9. The temperature value of the cold and ambient load is taken from the thermometer testo 735 .

Based on the K-band test platform, we have carried out 5 sets of tests for the cold and ambient load method in the laboratory. Table 2 provides the test data and calculation results of the cold and ambient load method and the comparison error with the theoretical calculation noise temperature $(406.5 \mathrm{~K})$.

3.3. Chopper Wheel Method. The K-band chopper wheel calibration platform is composed of a K-band ambient receiver, platform frame, elevation adjustment mechanism, and ambient blackbody load chopper mechanism. The platform can adjust the K-band receiver beam elevation angle at 0-90 degrees and realize ambient load chopper calibration function. The pictures of the K-band chopper 


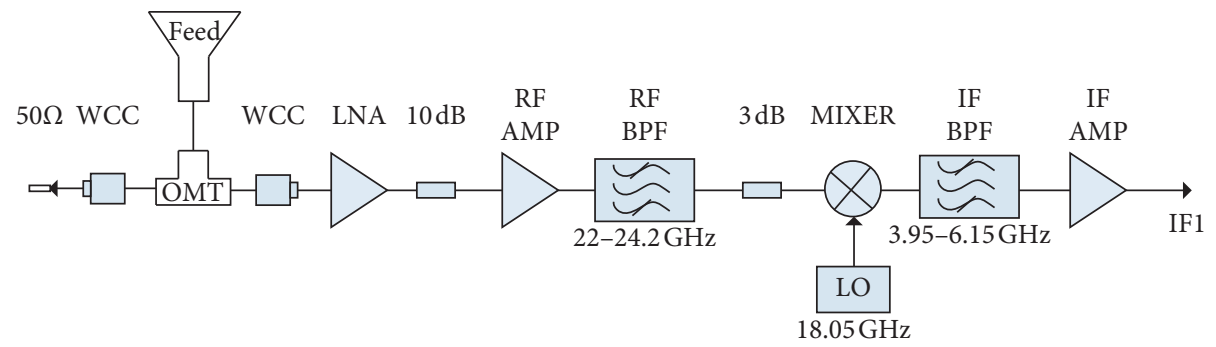

Figure 6: Structure of the K-band ambient receiver.

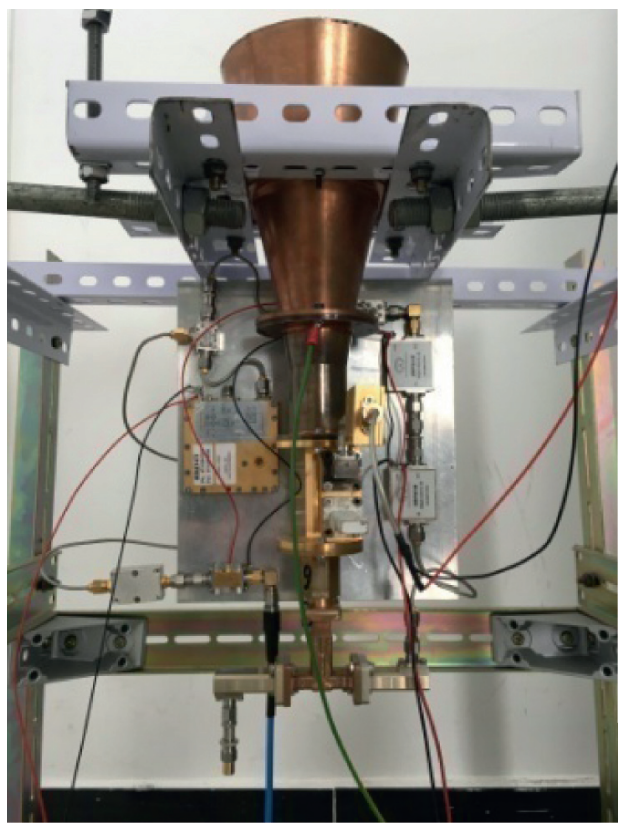

FIgURE 7: Picture of K-band ambient receiver.

TABLE 1: Theoretical calculation of noise temperature for the K-band receiver.

\begin{tabular}{|c|c|c|c|c|c|}
\hline Frequency $(\mathrm{GHz})$ & Gain $(\mathrm{dB})$ & Ambient $T(\mathrm{~K})$ & Noise figure $(\mathrm{dB})$ & Noise $T(\mathrm{~K})$ & Efficiency $T(\mathrm{~K})$ \\
\hline Mylar & -0.05 & 295 & 0.050 & 3.4 & 3.4159 \\
\hline Feed & -0.2 & 295 & 0.200 & 13.9 & 14.0639 \\
\hline OMT & -0.07 & 295 & 0.070 & 4.8 & 5.0774 \\
\hline WCC & -0.4 & 295 & 0.400 & 28.5 & 30.6373 \\
\hline LNA & 30 & 295 & 3.000 & 293.6 & 346.5450 \\
\hline $10 \mathrm{~dB}$ & -10 & 295 & 10.000 & 2655.0 & 3.1338 \\
\hline Amp & 30 & 295 & 3.000 & 293.6 & 3.4654 \\
\hline $\mathrm{BPF}$ & -0.7 & 295 & 0.700 & 51.6 & 0.0006 \\
\hline $3 \mathrm{~dB}$ & -3 & 295 & 3.000 & 293.6 & 0.0041 \\
\hline Mixer & -8 & 295 & 8.000 & 1566.3 & 0.0433 \\
\hline $\mathrm{BPF}$ & -0.5 & 295 & 0.500 & 36.0 & 0.0063 \\
\hline Amp & 30 & 295 & 3.000 & 293.6 & 0.0575 \\
\hline
\end{tabular}

calibration platform and ambient load are shown in Figure 10.

Based on the K-band chopper wheel test platform, we carried out 5 sets of tests for the chopper wheel method at Nanshan observation station at 90, 30, and 5 degrees elevation, respectively, in clear night. Table 3 provides the test data and calculation results of the chopper wheel method and the comparison error with the theoretical calculation noise temperature $(381.7 \mathrm{~K})$. Among them, the K-band sky brightness temperature is taken from the test data of the water vapor radiometer at $23.8 \mathrm{GHz}$ at Nanshan observation station; when the elevation is 90,30 , and 5 degrees, the $\mathrm{K}$-band sky brightness temperature is taken as $27.2 \mathrm{~K}, 39.5 \mathrm{~K}$, and $187.1 \mathrm{~K}$, respectively. 


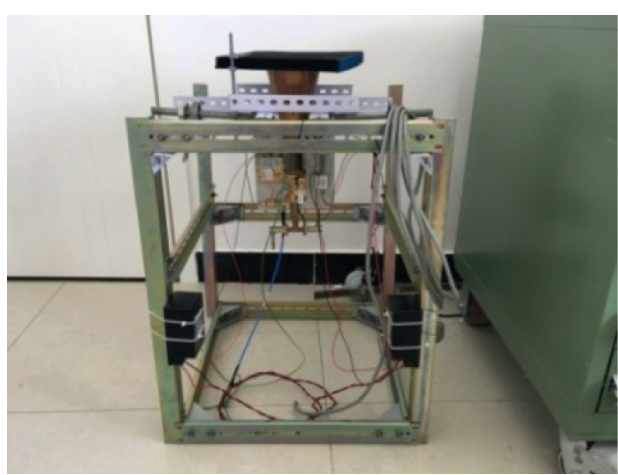

(a)

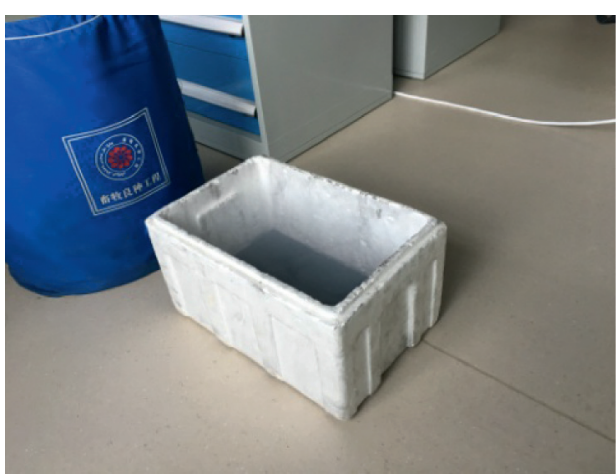

(b)

Figure 8: K-band test platform and cold load.

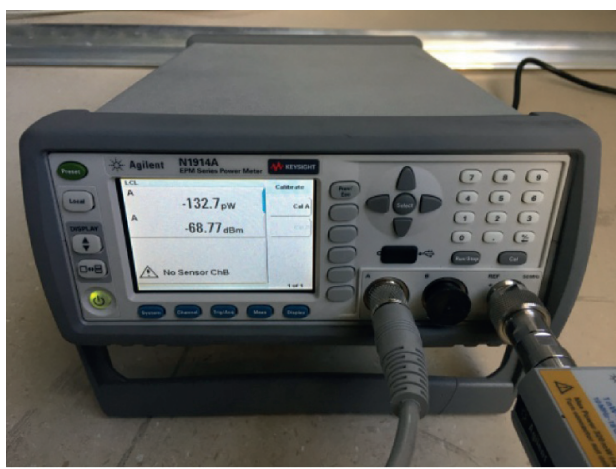

(a)

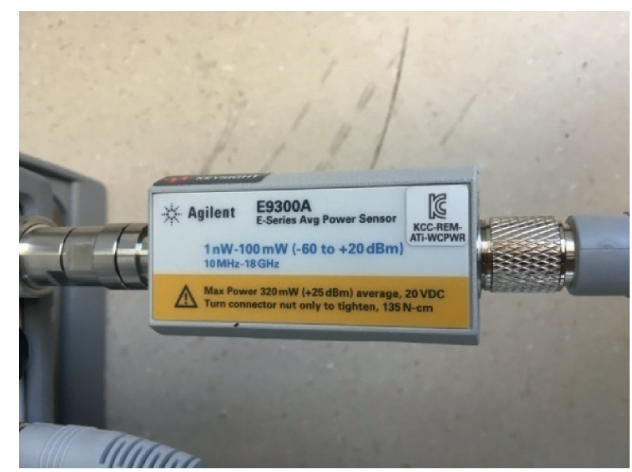

(b)

Figure 9: Agilent power meter and power probe.

TABLE 2: Test data and comparison results for the cold and ambient load method.

\begin{tabular}{lccccc}
\hline$T_{\mathrm{amb}}(\mathrm{K})$ & $V_{\mathrm{amb}}(\mathrm{uW})$ & $T_{\text {cold }}(\mathrm{K})$ & $V_{\text {cold }}(\mathrm{uW})$ & $T_{\text {rec }}(\mathrm{K})$ & 416.3 \\
\hline 295.7 & 66.77 & 77.9 & 46.35 & 417.4 & 2.4 \\
295.6 & 66.76 & 78 & 46.39 & 419.4 & 2.7 \\
295.6 & 66.78 & 78.2 & 46.48 & 418.8 \\
295.9 & 66.96 & 77.9 & 46.54 & 4.2 \\
295.2 & 66.98 & 78 & 46.72 & 422.7 & 3.0 \\
\hline
\end{tabular}

3.4. Test of the Ambient and Hot Load Method. On the basis of the cold and ambient load method and chopper wheel method, an ambient and hot load method is proposed, that is, by introducing the ambient and hot blackbody load alternately at the top of the feed, the receiver can test the radiation of the ambient and hot load alternately, so as to test the noise temperature of the receiver. The principle of this method is the same as that of the cold and ambient load method, but the cold load, which is not easy to realize, is replaced by the hot load. In the actual antenna observation, the ambient and hot load can be adjusted along with the beam direction of the receiver and can calibrate when the antenna is in any elevation angle in real time as the chopper wheel method.
Equation (10) is the noise temperature calculation formula of the receiver with the ambient and hot load method, wherein $T_{\text {hot }}$ is the temperature value of the hot load, and $T_{\text {hot }}$ is the corresponding power output of the receiver with the hot load placed on the feed interface.

$$
T_{\text {rec }}=\frac{T_{\text {hot }}-\left(V_{\text {hot }} / V_{\mathrm{amb}}\right) T_{\mathrm{amb}}}{\left(V_{\mathrm{hot}} / V_{\mathrm{amb}}\right)-1 .}
$$

The design of hot load is shown in Figure 11. This load is a flat blackbody produced by Nanjing Kepin Electronic Technology Co., Ltd., and it adopts a two-layer design structure, and the reflection coefficient of vertical incidence of the blackbody is $30 \mathrm{~dB}$ (at $18 \mathrm{GHz}$ ) and $35 \mathrm{~dB}$ (at $40 \mathrm{GHz}$ ), which fully meets the test requirements of K-band. The 


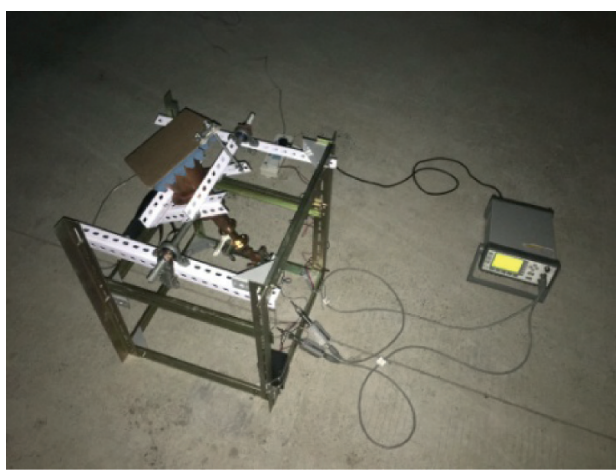

(a)

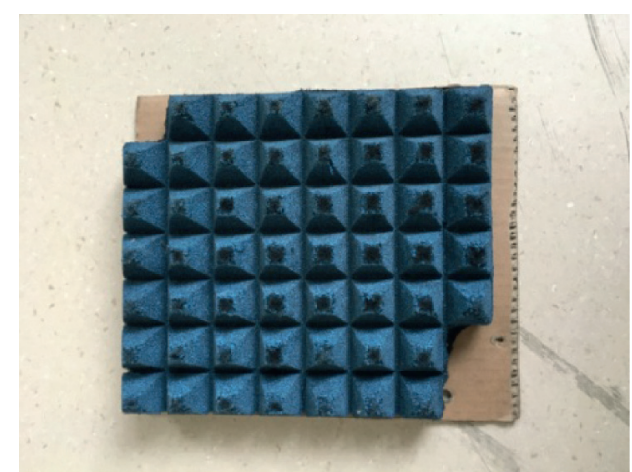

(b)

FIGURE 10: K-band chopper calibration platform and ambient load.

TABle 3: Test data and comparison results at 90, 30, and 5 degrees elevation.

\begin{tabular}{|c|c|c|c|c|c|c|c|c|c|c|c|c|c|}
\hline $\begin{array}{l}T_{\mathrm{amb}} \\
(\mathrm{k})\end{array}$ & $\begin{array}{l}V \text { amb } \\
(\mathrm{uW})\end{array}$ & $\begin{array}{c}T_{\text {sky }} \\
(\mathrm{k})\end{array}$ & $\begin{array}{l}V_{\text {sky }} \\
(\mathrm{uW})\end{array}$ & $T_{\text {rec }}(\mathrm{k})$ & $\begin{array}{l}\text { E90 } \\
(\%) \\
\end{array}$ & $\begin{array}{c}T_{\text {sky }} \\
(\mathrm{k})\end{array}$ & $\begin{array}{r}V_{\text {sky }} \\
(\mathrm{uW})\end{array}$ & $T_{\text {rec }}(\mathrm{k})$ & $\begin{array}{l}\text { E30 } \\
(\%) \\
\end{array}$ & $T_{\text {sky }}(\mathrm{k})$ & $\begin{array}{r}V_{\text {sky }} \\
(\mathrm{uW})\end{array}$ & $T_{\text {rec }}(\mathrm{k})$ & $\begin{array}{l}\text { E5 } \\
(\%) \\
\end{array}$ \\
\hline 277.8 & 97.65 & 27.2 & 61.21 & 393.7 & 3.1 & 39.5 & 63.01 & 394 & 3.2 & 187.1 & 85.7 & 463.4 & 21.4 \\
\hline 277.4 & 96.53 & 27.2 & 60.72 & 397 & 4 & 39.5 & 63.18 & 411.2 & 7.7 & 187.1 & 85.28 & 497.4 & 30.3 \\
\hline 276.7 & 95.5 & 27.2 & 60.23 & 395.9 & 3.7 & 39.5 & 62.38 & 407.3 & 6.7 & 187.1 & 84.28 & 485.9 & 27.3 \\
\hline 276.1 & 97.03 & 27.2 & 60.45 & 384.1 & 0.6 & 39.5 & 63.38 & 406.1 & 6.4 & 187.1 & 85.75 & 489.5 & 28.2 \\
\hline 276.5 & 96.08 & 27.2 & 60.42 & 395.2 & 3.5 & 39.5 & 62.89 & 409.6 & 7.3 & 187.1 & 84.92 & 493.2 & 29.2 \\
\hline
\end{tabular}

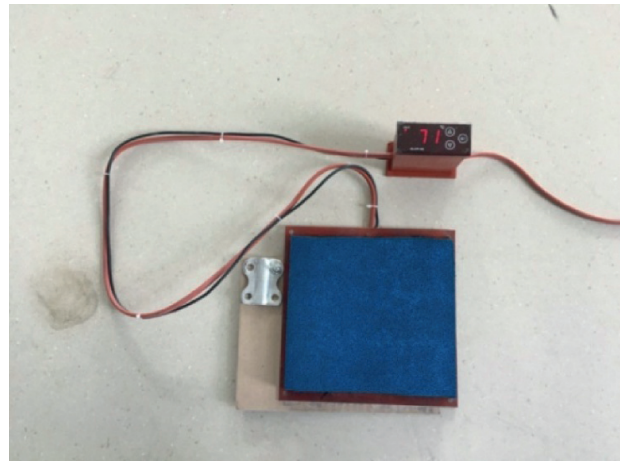

Figure 11: Design of the hot blackbody load.

heating resistance wire is evenly arranged in a $25 * 20 \mathrm{~cm}$ heating plate, and a temperature sensor is installed in the middle of the heating plate, with an LED digital monitoring meter, which can heat up to $150^{\circ} \mathrm{C}$. We paste an $18 * 18 \mathrm{~cm}$ blackbody on the lower part of the heating plate, and install it on the rotating shaft together with the chopper blade, so as to remove the hot blackbody from the feed port surface after the calibration test. When the heating function is turned on, the LED digital monitoring meter displays the current heating plate temperature. Set the heating plate to be heated to $120^{\circ} \mathrm{C}$ and then stop heating. When the temperature of the heating plate drops to $110^{\circ} \mathrm{C}$, turn on the heating function again. After heating to $120^{\circ} \mathrm{C}$, stop heating, and then cycle.

At the time of calibration, first place the ambient load at the front stage of the receiver's feed, collect the ambient load temperature and amplitude value of the power meter's corresponding output; then place the heated hot load $\left(120^{\circ} \mathrm{C}\right)$ at the front stage of the feed, collect the hot load temperature and amplitude value of power meter's corresponding output, and then calculate the receiver's noise temperature. Figure 12 shows the calibration of the ambient load at $90^{\circ}$ elevation on the left, and Figure 12 shows the calibration of the hot load at $90^{\circ}$ elevation on the right.

Based on the K-band ambient and hot load method test platform, we have carried out 5 sets of tests for the ambient and hot load method in the laboratory. Table 4 provides the test data and calculation results of the ambient and hot load method, as well as the comparison error with the theoretical calculation noise temperature.

\section{Discussion}

According to the above test results, the test error of the classical cold and ambient load method is between $2.4 \%$ and $4 \%$. Under the premise that the sky temperature can be accurately obtained by the chopper wheel method, the error of $90^{\circ}$ elevation is between $0.6 \%$ and $4 \%$, the error of $30^{\circ}$ elevation is between $3.2 \%$ and $7.7 \%$, and the error of $5^{\circ}$ elevation is between $21.4 \%$ and $30.3 \%$. The test error of the ambient and hot load method is between $0.9 \%$ and $10.7 \%$.

No matter which noise temperature calibration method is adopted, the test results are susceptible to port loss, temperature change, external electromagnetic interference, random jitter, and other factors. In order to ensure the validity of the test data, accurate measurement and evaluation of the accuracy of the noise test system are the basis for the analysis of its error sources [19].

Taking the ambient and hot load method to calibrate the receiver noise temperature as an example, the factors that 


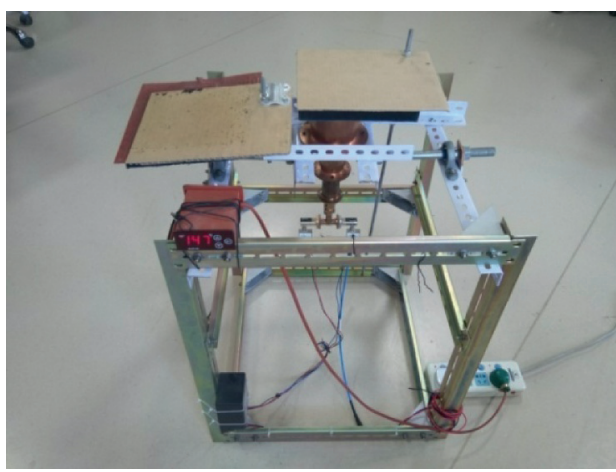

(a)

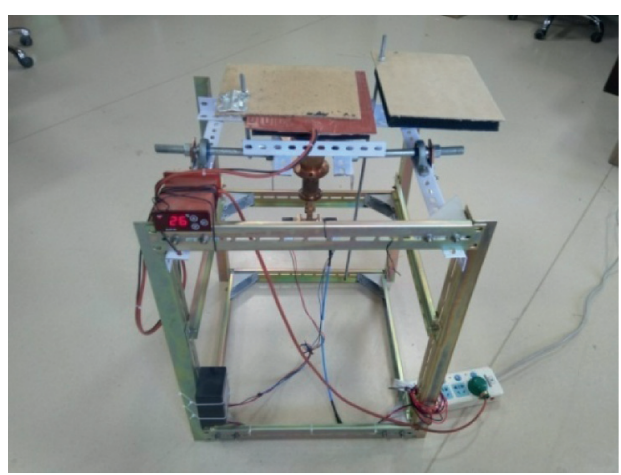

(b)

FigURE 12: K-band ambient and hot load calibration system.

TABLE 4: Test data and comparison results at 90, 30, and 5 degrees elevation.

\begin{tabular}{lccccc}
\hline$T_{\mathrm{amb}}(\mathrm{K})$ & $V_{\mathrm{amb}}(\mathrm{uW})$ & $T_{\text {hot }}(\mathrm{K})$ & $V_{\text {hot }}(\mathrm{uW})$ & $T_{\text {rec }}(\mathrm{K})$ & $E(\%)$ \\
\hline 293.1 & 66.29 & 388.7 & 75.73 & 378.1 & 7.0 \\
293.6 & 66.8 & 383.6 & 75.34 & 410.2 & 0.9 \\
294.1 & 66.91 & 388.2 & 75.58 & 431.9 & 6.3 \\
294.3 & 67.04 & 383.2 & 75.19 & 436.8 & 7.5 \\
294.4 & 66.92 & 385.3 & 75.09 & 450 & 10.7 \\
\hline
\end{tabular}

introduce the error of the receiver noise temperature are the four parameters on the right side of equation (10), that is, the calibration accuracy is related to the four parameters of the test, that is, the temperature of the ambient and hot load and the corresponding amplitude output $\left(T_{\mathrm{amb}}, T_{\mathrm{hot}}, V_{\mathrm{amb}}\right.$, and $\left.V_{\text {hot }}\right)$. Since the errors introduced by each parameter are independent of each other, the variance of $T_{\text {rec }}$ in this method is shown in equation (11):

$$
\begin{aligned}
\sigma^{2}\left(T_{\mathrm{rec}}\right)= & \sigma^{2}\left(T_{\mathrm{hot}}\right)\left(\frac{\partial T_{\mathrm{rec}}}{\partial T_{\mathrm{hot}}}\right)^{2}+\sigma^{2}\left(T_{\mathrm{amb}}\right)\left(\frac{\partial T_{\mathrm{rec}}}{\partial T_{\mathrm{amb}}}\right)^{2} \\
& +\sigma^{2}\left(V_{\mathrm{hot}}\right)\left(\frac{\partial T_{\mathrm{rec}}}{\partial V_{\mathrm{hot}}}\right)^{2}+\sigma^{2}\left(V_{\mathrm{amb}}\right)\left(\frac{\partial T_{\mathrm{rec}}}{\partial V_{\mathrm{amb}}}\right)^{2} .
\end{aligned}
$$

In the receiver noise temperature $T_{\text {rec }}$, partial derivatives of $T_{\text {hot }}, T_{\mathrm{amb}}, V_{\mathrm{amb}}$, and $V_{\text {hot }}$ were obtained, respectively, as shown in equations (12)-(15):

$$
\begin{aligned}
\frac{\partial T_{\mathrm{rec}}}{\partial T_{\mathrm{hot}}} & =\frac{V_{\mathrm{amb}}}{V_{\mathrm{hot}}-V_{\mathrm{amb}}}, \\
\frac{\partial T_{\mathrm{rec}}}{\partial T_{\mathrm{amb}}} & =\frac{V_{\mathrm{hot}}}{V_{\mathrm{amb}}-V_{\mathrm{hot}}}, \\
\frac{\partial T_{\mathrm{rec}}}{\partial V_{\mathrm{amb}}} & =\frac{V_{\mathrm{hot}}\left(T_{\mathrm{hot}}-T_{\mathrm{amb}}\right)}{\left(V_{\mathrm{hot}}-V_{\mathrm{amb}}\right)^{2}}, \\
\frac{\partial T_{\mathrm{rec}}}{\partial V_{\mathrm{hot}}} & =\frac{V_{\mathrm{amb}}\left(T_{\mathrm{amb}}-T_{\mathrm{hot}}\right)}{\left(V_{\mathrm{hot}}-V_{\mathrm{amb}}\right)^{2}} .
\end{aligned}
$$

After that, the variance of each parameter can be obtained according to equation (16) by combining the test data of several groups of four parameters, wherein $\sigma^{2}$ is the population variance, $X_{i}$ is the test value of a group, $\bar{X}$ is the test mean of many groups, and $\mathrm{N}$ is the number of test groups.

$$
\sigma^{2}=\frac{\sum\left(X_{i}-\bar{X}\right)^{2}}{N}
$$

Finally, the precision calculation of the ambient and hot load method is shown in equation (17):

$$
\frac{\sigma\left(T_{\mathrm{rec}}\right)}{T_{\mathrm{rec}}}=\sqrt{\sigma^{2}\left(T_{\mathrm{hot}}\right)\left(\frac{V_{\mathrm{amb}}}{V_{\mathrm{hot}}-V_{\mathrm{amb}}}\right)^{2}+\sigma^{2}\left(T_{\mathrm{amb}}\right)\left(\frac{V_{\mathrm{hot}}}{V_{\mathrm{amb}}-V_{\mathrm{hot}}}\right)^{2}+\sigma^{2}\left(V_{\mathrm{hot}}\right)\left(\frac{V_{\mathrm{amb}}\left(T_{\mathrm{amb}}-T_{\mathrm{hot}}\right)}{\left(V_{\mathrm{hot}}-V_{\mathrm{amb}}\right)^{2}}\right)^{2}+\sigma^{2}\left(V_{\mathrm{amb}}\right)\left(\frac{V_{\mathrm{hot}}\left(T_{\mathrm{hot}}-T_{\mathrm{amb}}\right)}{\left(V_{\mathrm{hot}}-V_{\mathrm{amb}}\right)^{2}}\right)^{2}}
$$

According to the temperature and power test data of 5 groups of the ambient and hot load method listed in Table 4, and substituted into equation (16), we can get that $\sigma^{2}\left(T_{\mathrm{amb}}\right)$ is $0.236, \sigma^{2}\left(V_{\mathrm{amb}}\right)$ is $0.069, \sigma^{2}\left(T_{\text {hot }}\right)$ is 5.204 , and $\sigma^{2}\left(V_{\text {hot }}\right)$ is 0.057 , respectively. After that, each parameter obtained is substituted into equation (17), respectively, and the accuracy of each group of tests of the ambient and hot load method can be obtained, as shown in Table 5 .

Similarly, according to the test data of 5 groups of cold and ambient load temperature and power listed in Table 2, and according to equation (16), we can get that $\sigma^{2}\left(T_{\mathrm{amb}}\right)$ is $0.052, \sigma^{2}\left(V_{\mathrm{amb}}\right)$ is $0.01, \sigma^{2}\left(T_{\text {cold }}\right)$ is 0.012 , and $\sigma^{2}\left(V_{\text {cold }}\right)$ is 
TABLE 5: Accuracy calculation results of the ambient and hot load method.

\begin{tabular}{lccc}
\hline$T_{\text {rec }}(\mathrm{K})$ & $\sigma^{2}\left(T_{\text {rec }}\right)$ & $\sigma\left(T_{\text {rec }}\right)$ & $\sigma\left(T_{\text {rec }}\right) / T_{\text {rec }}$ \\
\hline 406.5 & 1015.4977 & 31.8669 & 7.84 \\
406.5 & 1320.52 & 36.339 & 8.94 \\
406.5 & 1345.468 & 36.68 & 9.02 \\
406.5 & 1529.8874 & 39.1138 & 9.62 \\
406.5 & 1564 & 39.5474 & 9.73 \\
\hline
\end{tabular}

0.017. Then, each parameter can be substituted according to equation (17), and the accuracy of each group of tests of the cold and ambient load method can be obtained, as shown in Table 6.

As the sky brightness temperature is directly determined by the water vapor radiometer, taking the chopper wheel method of $90^{\circ}$ elevation as an example, the test data of 5 groups of ambient load temperature and power and sky intensity are listed in Table 3; according to equation (16), it can be obtained as $\sigma^{2}\left(T_{\mathrm{amb}}\right)$ is $0.38, \sigma^{2}\left(V_{\mathrm{amb}}\right)$ is 0.5528 , and $\sigma^{2}\left(V_{\text {sky }}\right)$ is 0.1156 . Then, each parameter can be substituted according to equation (17), and the accuracy of each test group of the chopper method at $90^{\circ}$ elevation angle can be obtained, as shown in Table 7.

From the final test results, the test accuracy of the ambient and hot load method is between $7.84 \%$ and $9.73 \%$, the test accuracy of the cold and ambient load method is between $1.28 \%$ and $1.3 \%$, and the test accuracy of the chopper wheel method is between $2.73 \%$ and $2.92 \%$ at $90^{\circ}$ elevation, between $3 \%$ and $3.22 \%$ at $30^{\circ}$ elevation, and between $13.86 \%$ and $15.47 \%$ at $5^{\circ}$ elevation. Analysis reason and the ambient and hot load method variance of four parameters were higher than the cold and ambient load method of the calculated results, especially the variance of hot load temperature is almost two orders of magnitude higher than the cold and ambient method; the judgment and high temperature of the uncertainty of the hot load which lead to the deterioration of the accuracy of this method are one of the main reasons. On the other hand, as shown in equation (17), the temperature and power difference between the two loads of each method will also directly affect the calibration accuracy, which is another reason why the test accuracy of the ambient and hot load method is inferior to that of the cold and ambient load method, and the chopper wheel method, while the temperature and the power difference are bigger than the other methods, combined with the sky brightness temperature for a water vapor radiometer given a fixed value, makes the precision of calculation be the highest in theory; but as a result of the test ambient load radiation and cold sky radiation power, output in the outdoor environment is not stable, corresponding to the variance is nearly an order of magnitude higher than that of indoor environment testing, makes the final accuracy lower than the cold and ambient load method, and is $5^{\circ}$ in low elevation due to the introduction of the ground noise that accuracy is the further deterioration.
TABLE 6: Accuracy calculation results of the cold and ambient load method.

\begin{tabular}{lccc}
\hline$T_{\text {rec }}(\mathrm{K})$ & $\sigma^{2}\left(T_{\text {rec }}\right)$ & $\sigma\left(T_{\text {rec }}\right)$ & $\sigma\left(T_{\text {rec }}\right) / T_{\text {rec }}(\%)$ \\
\hline 406.5 & 26.9353 & 5.1899 & 1.28 \\
406.5 & 27.154 & 5.21 & 1.28 \\
406.5 & 27.515 & 5.2455 & 1.29 \\
406.5 & 27.1533 & 5.2109 & 1.28 \\
406.5 & 27.8745 & 5.2796 & 1.30 \\
\hline
\end{tabular}

TABLE 7: Accuracy calculation results of the chopper wheel method at $90^{\circ}$.

\begin{tabular}{lccc}
\hline$T_{\text {rec }}(\mathrm{K})$ & $\sigma^{2}\left(T_{\text {rec }}\right)$ & $\sigma\left(T_{\text {rec }}\right)$ & $\sigma\left(T_{\text {rec }}\right) / T_{\text {rec }}(\%)$ \\
\hline 381.7 & 114.1 & 10.68 & 2.8 \\
381.7 & 119.69 & 10.94 & 2.87 \\
381.7 & 124.2 & 11.14 & 2.92 \\
381.7 & 108.6 & 10.42 & 2.73 \\
381.7 & 119.67 & 10.94 & 2.86 \\
\hline
\end{tabular}

Similarly, Tables 8 and 9 show the test accuracy of each group of the chopper wheel method at $30^{\circ}$ and $5^{\circ}$ elevation angles.

TABLE 8: Accuracy calculation results of the chopper wheel method at $30^{\circ}$.

\begin{tabular}{lccc}
\hline$T_{\text {rec }}(\mathrm{K})$ & $\sigma^{2}\left(T_{\text {rec }}\right)$ & $\sigma\left(T_{\text {rec }}\right)$ & $\sigma\left(T_{\text {rec }}\right) / T_{\text {rec }}(\%)$ \\
\hline 381.7 & 130.56 & 11.43 & 3 \\
381.7 & 150.77 & 12.28 & 3.22 \\
381.7 & 150.4 & 12.26 & 3.21 \\
381.7 & 145 & 12.04 & 3.15 \\
381.7 & 151.13 & 12.29 & 3.22 \\
\hline
\end{tabular}

TABLE 9: Accuracy calculation results of the chopper wheel method at $5^{\circ}$.

\begin{tabular}{lccc}
\hline$T_{\text {rec }}(\mathrm{K})$ & $\sigma^{2}\left(T_{\text {rec }}\right)$ & $\sigma\left(T_{\text {rec }}\right)$ & $\sigma\left(T_{\text {rec }}\right) / T_{\text {rec }}(\%)$ \\
\hline 381.7 & 2798.03 & 52.9 & 13.86 \\
381.7 & 3474.95 & 58.95 & 15.44 \\
381.7 & 3380.54 & 58.14 & 15.23 \\
381.7 & 3376.6 & 58.11 & 15.22 \\
381.7 & 3486.44 & 59.05 & 15.47 \\
\hline
\end{tabular}

\section{Conclusions}

In this paper, through the establishment of the K-band ambient receiver and its cold and ambient load method, chopper wheel method, and ambient and hot load method amplitude calibration test platform, comparison and analysis of the above three methods are carried out. The results show that the test accuracy of the cold and ambient load method is about $1.3 \%$, the test accuracy of the chopper method (under 90 or 30 degrees of elevation) is about $3 \%$, and the test accuracy of the ambient and hot load method is about $9 \%$.

As the most classical method of amplitude calibration, the cold and ambient load method has the highest test accuracy (test error within $4 \%$ ), but its disadvantage is that it 
cannot be carried out at any time during the observation process.

The chopper wheel method performed at any time during the process of observation, in the actual test receiver power output fluctuations due to the outdoor environment, makes the precision slightly lower than the traditional method of low room temperature load $\left(90^{\circ}\right.$ test error within $4 \%$ and $30^{\circ}$ test error within $7.7 \%$ ), and it will introduce ground noise under low elevation difference as bigger error ( $5^{\circ}$ test error within $30.3 \%$ ) and cannot be used; if corresponding to the sky bright temperature acquisition, introducing an additional variable will worsen further testing precision.

The ambient and hot load method of the test accuracy is slightly lower than that of the above two methods. The analysis is mainly due to the uncertainty of the high temperature black body temperature and the small temperature difference between the two loads, which leads to the deterioration of the overall accuracy. But the advantage is that the method can perform real-time calibration in the process of observation, and the method is easier to implement than the traditional cold and ambient load method. The results of noise temperature measurement are compared with those of theoretical calculation; the error is basically within 10\%; and it can satisfy the demand of the noise temperature test. In the future, we expect that on the basis of increasing the hot load temperature, further experiments were carried out on the thermostatic treatment of the hot load and the accuracy of temperature acquisition, and finally, we hope that this method can better meet the testing requirements of receiver noise temperature and radio source amplitude calibration.

\section{Data Availability}

The data used to support the findings of this study are available from the corresponding author upon request.

\section{Conflicts of Interest}

The authors declare that there are no conflicts of interest regarding the publication of this paper.

\section{Acknowledgments}

This work was supported by Xinjiang Key Laboratory of Radio Astrophysics (Microwave Technology) (No. 2015KL012), the National Natural Science Foundation of China (Nos. 11973078, 11903077, and 11603064), and the Natural Science Foundation of Xinjiang Uygur Autonomous Region (No. 2019D01A99). The work was partly supported by the Operation, Maintenance, and Upgrading Fund for Astronomical Telescopes and Facility Instruments, budgeted from the Ministry of Finance of China (MOF) and administrated by the Chinese Academy of Sciences.

\section{References}

[1] N. Wang, "Xinjiang Qitai $110 \mathrm{~m}$ radio telescope," Scientia Sinica Physica, Mechanica \& Astronomica, vol. 44, no. 8, pp. 783-794, 2014.
[2] M. Z. Chen, Q. Liu, J. Ma et al., "Ultra-wideband receiver technology development for the radio astronomical large aperture telescope," Scientia Sinicia-Physica Mechnica Astronomica, vol. 47, no. 5, Article ID 059504, 2017.

[3] B. B. Xiang, C. S. Wang, W. Wang et al., "Adjustment method of subreflector position of reflector antennas based on the electromechanical couple theory," Systems Engineering and Electronic Technology, vol. 40, no. 3, pp. 489-497, 2018.

[4] J. Ma, X. Pei, N. Wang et al., "Ultra-wideband and multibeam signal receiving and processing system of QTT," Scientia Sinicia-Physica Mechnica Astronomica, vol. 49, no. 9, Article ID 099502, 2019.

[5] K. Wang, M. Z. Chen, J. Ma et al., "The amplitude calibration of radio astronomy millimeter wave receiver," Acta Astronomica Sinica, vol. 59, no. 5, pp. 1-14, 2018.

[6] K. Z. Han, X. Geng, F. Zhang et al., "Discussion on vertex problem of the black-body radiation curve," Physics Bulletin.vol. 37, no. 10, pp. 115-118, 2018.

[7] K. Wang, X. F. Li, M. Z. Chen et al., "Measurements and analyses of noise temperature for the $\mathrm{Ku}$ band receiver," Electro-mechanical Engineering, vol. 34, no. 6, pp. 13-16, 2018.

[8] A. A. Penzias and C. A. Burrus, "Millimeter-wavelength radioastronomy techniques," Annual Review of Astronomy and Astrophysics, vol. 11, no. 1, pp. 51-72, 1973.

[9] B. L. Ulich and R. W. Haas, "Absolute calibration of millimeter-wavelength spectral lines," The Astrophysical Journal Supplement Series, vol. 30, pp. 247-258, 1976.

[10] B. L. Ulich, "Millimeter-wavelength continuum calibration sources," The Astronomical Journal, vol. 86, no. 86, pp. 1619-1626, 1981.

[11] X. D. Chen, Z. Y. Zhang, S. J. Lin et al., " $8 \mathrm{~mm}$ microwave sky temperature," Journal of Huazhong Insti-Tute of Technology, vol. 04, pp. 107-112, 1985.

[12] L. Q. Gui, Z. Y. Zhang, and W. Guo, "Calculation of sky brightness temperature in a $3 \mathrm{~mm}$ band," Journal of Huazhong University of Science and Technology: Natural Science Edition, vol. 12, pp. 79-81, 2005.

[13] B. Q. Lao, J. Q. Wang, J. Y. Wang et al., "Calculation of sky brightness temperature at 0.1-50 GHz," An-nual Journal of Shanghai Observatory, Chinese Academy of Sciences, vol. 1, pp. 21-31, 2014.

[14] J. Yang, Y. T. Cao, and S. F. Huang, "Measurement of the opacity of the earth's atmosphere at $97.8 \mathrm{GHz}$," Acta Astronomica Sinica, vol. 03, pp. 333-336, 1998.

[15] M. F. Zhou, Q. J. Yao, S. Li et al., "Measurement of $460 \mathrm{GHz}$ atmospheric opacity in Yangbajing," Acta Astronomica Sinica, vol. 01, pp. 64-74, 2011.

[16] P. R. Jewell, "Single-dish radio astronomy: techniques and applications," ASP Conference Series, vol. 278, pp. 313-328, 2002.

[17] J. Yang, "Atmospheric attenuation and intensity calibration of millimeter wave and submillimeter wave observations," Chinese Astronomy and Astrophysics, vol. 23, no. 2, p. 261, 1999.

[18] R. P. Meys, "A wave approach to the noise properties of linear microwave devices," IEEE Transactions on Microwave Theory and Techniques, vol. 26, no. 1, pp. 34-37, 1978.

[19] S. S. Peng and X. G. Li, "Study on calibration method of a $8 \mathrm{~mm}$ radiometer," Journal of Infrared and Millimeter Wave, vol. 04 , pp. 40-45, 1997. 